\title{
Efficacy of and Resistance to Rifaximin-based Quadruple Therapy in Helicobacter pylori Eradication
}

\author{
Hyun Soo Kim ${ }^{1, *}$, Hyuk Yoon ${ }^{1, *}$, Dong Woo Shin ${ }^{1}$, Dong Jun Oh ${ }^{1}$, Mingu Kwon ${ }^{1}$, Yoon Jin Choi ${ }^{1}$, Cheol Min Shin ${ }^{1}$, Young Soo Park , \\ Nayoung $\mathrm{Kim}^{1,2}$, Dong Ho Lee ${ }^{1,2}$ \\ Department of Internal Medicine, Seoul National University Bundang Hospital ${ }^{1}$, Seongnam, Department of Internal Medicine and Liver \\ Research Institute, Seoul National University College of Medicine ${ }^{2}$, Seoul, Korea
}

\begin{abstract}
Background/Aims: The treatment options for Helicobacter pylori (H. pylori) infection are in a state of flux: traditional triple therapies have started to fail, and new treatments are unable to achieve optimal eradication rates. Rifaximin and rifabutin are new antibiotics. The aim of this study was to evaluate the efficacy and safety of adding rifaximin to the standard triple regimen and of a rifabutin-based triple regimen as a rescue therapy for $H$. pylori eradication.

Materials and Methods: We enrolled $27 \mathrm{H}$. pylori-positive patients who were treated with a proton pump inhibitor, amoxicillin, clarithromycin, and rifaximin for 14 days. H. pylori eradication was assessed by a ${ }^{13} \mathrm{C}$-urea breath test performed 4 weeks after therapy completion. The efficacy of the therapy was based on intention-to-treat (ITT) and per-protocol (PP) analysis. We also investigated the resistance rate, compliance, and side effects associated with rifaximin therapy. Minimal inhibitory concentrations and resistance to rifabutin were evaluated using the agar dilution method.

Results: Of the 27 patients, 22 completed the treatment protocol with 100\% compliance; five patients withdrew. The ITT and PP eradication rates for the rifaximin-containing quadruple therapy were $70.4 \%(19 / 27)$ and $86.3 \%(19 / 22)$, respectively. Adverse events were observed in five of 22 patients (22.7\%). The resistance rates to rifaximin and rifabutin were $66.7 \%(2 / 3)$ and $0 \%(0 / 3)$, respectively.

Conclusions: The findings of this study show the limitations of rifaximin-based quadruple therapy and suggest the benefits of a rifabutin-based rescue regimen in South Korea. (Korean J Helicobacter Up Gastrointest Res 2020;20:218-224)
\end{abstract}

Key Words: Amoxicillin; Clarithromycin; Helicobacter pylori; Rifaximin

\section{INTRODUCTION}

Helicobacter pylori (H. pylori) is a gram-negative, microaerophilic bacterium that is common worldwide., ${ }^{1,2}$ For more than 30 years, H. pylori has been studied for its role in the development of gastrointestinal diseases, such as chronic gastritis, peptic ulcers, gastric mucosa-associated lymphoid tissue lymphoma, and gastric cancer. It is also categorized as a class I carcinogen by the World Health Organization. ${ }^{3,4}$ In addition, H. pylori is known to be associated with extra-gastrointestinal disorders such as urticaria, idiopathic thrombocytopenic purpura, acne rosacea, migraines, and glaucoma. ${ }^{5-10}$ Therefore, the success of $H$. pylori eradication is very

Received: March 20, 2020 Revised: April 22, 2020 Accepted: April 30, 2020 Corresponding author: Dong Ho Lee

Department of Internal Medicine, Seoul National University Bundang Hospital, 82 Gumi-ro 173beon-gil, Bundang-gu, Seongnam 13620, Korea

Tel: +82-31-787-7009, Fax: +82-31-787-4051, E-mail: dhljohn@yahoo.co.kr

*These authors contributed equally to this work.

This study was supported by SNUBH Research Fund (grant no. 02-2013-033). important for the prevention of disease progress.

The known classic triple regimen, consisting of a protonpump inhibitor (PPI) with two antibiotics (clarithromycin and amoxicillin/metronidazole), has been considered as the gold standard for the eradication of $H$. pylori. ${ }^{11,12}$ However, the eradication rate of first-line treatment is only $70 \%$ to $85 \%$ and is following a decreasing trend due to antibiotic resistance. ${ }^{12-14}$ In the past years, new antibiotic regimens have been studied to overcome treatment failure. New macrolides, fluoroquinolones, and rifabutin have been used with various eradication rates achieved. ${ }^{15-17}$ Rifaximin, one of the among the new antibiotics, is a poorly absorbed synthetic rifamycin derivative, which has a wide spectrum of antimicrobial activity against both gram-positive and gram-negative aerobic and anaerobic bacteria. ${ }^{18}$ As it is poorly absorbed in the gastrointestinal tract, rifaximin has high bioavailability in the gastrointestinal tract. Therefore, it achieves intestinal concentrations that are 250 500 times

Copyright $\odot 2020$ Korean College of Helicobacter and Upper Gastrointestinal Research

(a) The Korean Journal of Helicobacter and Upper Gastrointestinal Research is an Open-Access Journal. All articles are distributed under the terms of the Creative Commons Attribution Non-Commercial License (http:// creativecommons.org/licenses/by-nc/4.0) which permits unrestricted non-commercial use, distribution, and reproduction in any medium, provided the original work is properly cited. 
higher than the minimal inhibitory concentration (MIC) 90. ${ }^{18,19}$ In in vitro studies, rifaximin was shown to inhibit H. pylori growth (MC50, $4 \mathrm{\mu g} / \mathrm{mL}$; MC90, $8 \mathrm{\mu g} / \mathrm{mL}$ ); thus, we believe it is a potential antibiotic for $H$. pylori eradication. Quesada et al. ${ }^{20}$ showed that rifaximin was useful against $H$. pylori strains resistant to clarithromycin ${ }^{21}$ and suggested that it may be effective in combination with the classic triple therapy or for treatment in patients who have failed previous eradication therapies with clarithromycin. It is known that rifaximin-based 7-day triple therapy (esomeprazole $40 \mathrm{mg}$ o.d., clarithromycin $500 \mathrm{mg}$ b.i.d., rifaximin $400 \mathrm{mg}$ t.i.d.) has a higher compliance rate than the standard triple therapy but a lower eradication rate. ${ }^{22}$ In addition, Choi et al..$^{23}$ showed that a rifaximin-based 7-day quadruple regimen (omeprazole 20 mg b.i.d., amoxicillin 1 g b.i.d., levofloxacin $200 \mathrm{mg}$ b.i.d., rifaximin $400 \mathrm{mg}$ b.i.d.) was not inferior to standard triple therapy in eradication rate and had fewer side effects. Therefore, the quadruple therapy, with the addition of rifaximin to the standard triple regimen, is believed to act against clarithromycin-resistant H. pylori strains and increase the eradication rate. In addition, rifabutin-based triple therapy, which includes a rifamycin derivative, has been suggested as a third-line treatment for $H$. pylori rescue therapy in Europe. However, there have been a few reports, except for retrospective studies, on small samples of patients in Korea. ${ }^{24}$ Therefore, the aim of this study was to evaluate the efficacy and safety of the addition of rifaximin to the standard triple regimen and the possibility of a rifabutin-based triple regimen as a rescue therapy for the eradication of $H$. pylori.

\section{MATERIALS AND METHODS}

\section{Patients}

We conducted this prospective pilot study between February 2014 and July 2015 at the Seoul National University Bundang Hospital in Korea. We enrolled 27 patients who were naïve to eradication treatment and were infected with H. pylori. H. pylori infection was defined as having at least one of the following: 1) histologic evidence of $H$. pylori, identified using modified Giemsa staining in the high body greater curvature; 2) a positive rapid urease test (CLO test; Delta West, Bentley, Australia), performed out using a gastric mucosa biopsy from the high body greater curvature; and 3) a positive result for ${ }^{13} \mathrm{C}$-urea breath test (UBT). We excluded the patients who were below 18 or above 80 years of age, pregnant women, patients with severe pulmonary, renal, or uncompensated liver cirrhosis or any other untreated malignancies. Patients who had taken PPIs, histamine-2 receptor antagonists, or antibiotics within 2 months prior to the trial were also excluded. The study was approved by the Institutional Review Board of the Seoul National University Bundang Hospital (IRB No: B-1308/215-009).

This study was conducted by following the ethical principles for medical research involving human subjects based on the Declaration of Helsinki. Furthermore, it has been approved by the Medical Ethics Committee and aspires to protect the lives, health, privacy, and dignity of research participants.

\section{Study design}

Patients who met the enrollment and exclusion criteria and signed the informed consent form were administered rifaximin $600 \mathrm{mg}$ b.i.d., amoxicillin 1,000 mg b.i.d., clarithromycin $500 \mathrm{mg}$ b.i.d., and a standard PPI dose (pantoprazole $40 \mathrm{mg}$, esomeprazole $40 \mathrm{mg}$, lansoprazole $30 \mathrm{mg}$, omeprazole $20 \mathrm{mg}$, rabeprazole $20 \mathrm{mg}$ before meals) b.i.d. as a first-line treatment for $H$. pylori infection. The treatment duration was 14 days. The patients were educated about the importance of full compliance, warned of the side effects, and provided a contact number of the hospital in case they encountered any problems. Two weeks after the end of the therapy, a post-treatment follow-up visit was scheduled to evaluate compliance and any associated side effects. Compliance was evaluated by direct questioning and by asking the patients to bring all the drugs that had been provided at the beginning of treatment so that they could be accounted for. Four weeks after the treatment was started, $H$. pylori eradication was then classified as one of the following: 1) no histologic evidence of $H$. pylori, identi- 
fied using modified Giemsa staining in the high body greater curvature; 2) a negative rapid urease test (CLO test; Delta West), measured using a gastric mucosa biopsy from the high body greater curvature; and 3) a negative result for ${ }^{13} \mathrm{C}$-UBT.

\section{3. ${ }^{13} \mathrm{C}-\mathrm{UBT}$}

Patients were required to fast for 4 hours before testing. No test meal was provided, and a pre-dose breath sample was obtained; $100 \mathrm{mg}$ of ${ }^{13} \mathrm{C}$-urea powder (UBiTkit; Otsuka Pharmaceutical Co. Ltd., Tokyo, Japan) dissolved in $100 \mathrm{~mL}$ of water was administered orally, and a second breath sample was collected $20 \mathrm{mi}^{-}$ nutes later. The cut-off value was $2.5 \%$. The collected samples were analyzed using an isotope ratio mass spectrometer (UBiT-IR300 ${ }^{\circledR}$; Otsuka Pharmaceutical Co. Ltd.). The patients were asked to avoid acid-lowering drugs or antibiotics for 1 week before the UBT.

\section{H. pylori strains and antibiotic susceptibility testing}

Three specimens were used for culture, and the susceptibility of isolated $H$. pylori strains to antibiotics (amoxicillin, clarithromycin, metronidazole, and rifaximin) was determined by using an agar dilution method. H. pylori strains previously obtained were cultured at $37^{\circ} \mathrm{C}$ on brain-heart infusion plates under micro-aerobic conditions $\left(5 \% \mathrm{O}_{2}, 10 \% \mathrm{CO}_{2}\right.$, and $\left.85 \% \mathrm{~N}_{2}\right)$ for $3 \sim 5$ days. H. pylori was identified based on Gram staining, colony morphology, and positive reactions to oxidase, catalase, and urease. We performed MIC tests for the previously mentioned antibiotics using a serial two-fold agar dilution method. The organisms were cultured on MuellerHinton agar supplemented with 5\% defibrinated sheep's blood for 48 hours. As we were testing the potential of rifabutin-containing rescue therapy after failure of first-line or second-line treatment, the MIC for rifabutin was also determined. The bacterial suspension, with $1 \times 10^{7}$ colony-forming units, was inoculated onto agar plates containing each antibiotic. We identified the MIC after incubation for 72 hours. According to the clinical breakpoints for $H$. pylori proposed by the European Committee on Antimicrobial Susceptibility Testing, the resistance breakpoints for each antibiotic were as follows: $0.12 \mathrm{mg} / \mathrm{L}$ for amoxicillin, $0.5 \mathrm{mg} / \mathrm{L}$ for clarithromycin, $8 \mathrm{mg} / \mathrm{L}$ for metronidazole, $1 \mathrm{mg} / \mathrm{L}$ for rifaximin, and $0.25 \mathrm{mg} / \mathrm{L}$ for rifabutin. ${ }^{25}$

\section{Statistical analysis}

The efficacy of $H$. pylori treatment was evaluated by the intention-to-treat (ITT) and per-protocol (PP) methods. ITT analysis compared the treatment groups that were initially allocated. In the case of PP, we excluded patients who were lost to follow-up, had taken less than $85 \%$ of the prescribed drugs, or had discontinued treatment due to severe adverse events.

\section{RESULTS}

\section{Patient population}

Twenty-seven consecutive patients (20 men and 7 women, mean age $55.5 \pm 13.8$ years) were included in this study. As the treatment was not a standard regimen, not many patients agreed to participate in the study. The indications for $H$. pylori eradication treatment were

Table 1. Baseline Demographics of the Patients

\begin{tabular}{lc}
\hline & $\begin{array}{c}\text { Rifaximin-based } \\
\text { quadruple therapy }\end{array}$ \\
\hline Included in ITT analysis & 27 \\
Male/female & $20 / 7$ \\
Age (years) & $55.5 \pm 13.8$ \\
Disease & \\
Benign gastric ulcer & $8(29.6)$ \\
Duodenal ulcer & $4(14.8)$ \\
Gastric dysplasia or cancer & $5(18.5)$ \\
Non-ulcer dyspepsia & $10(37.0)$ \\
Drop out & 5 \\
Noncompliance & $0(0)$ \\
Follow-up loss & $4(14.8)$ \\
Discontinued therapy because of adverse & $1(3.7)$ \\
$\quad$ events & \\
\hline
\end{tabular}

Values are presented as mean \pm standard deviation or $\mathrm{n}(\%)$. ITT, intention-to-treat. 
as follows: benign gastric ulcer (8, 29.6\%), duodenal ulcer $(4,14.8 \%)$, gastric dysplasia or cancer $(5,18.5 \%)$, and non-ulcer dyspepsia (10, 37\%). The characteristics of the study population are summarized in Table 1 . Of the 27 patients, 22 completed the treatment protocol; the re-

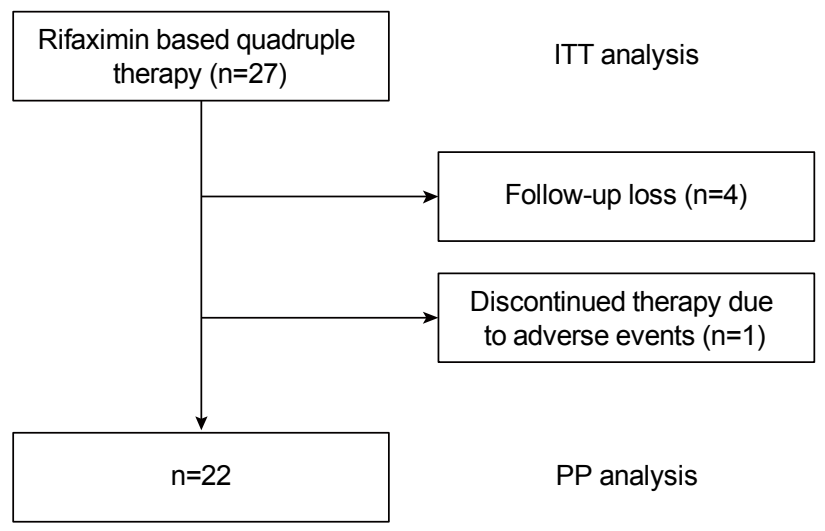

Fig. 1. Flow schematic of studies included in intention-to-treat (ITT) and per-protocol (PP) analysis.

Table 2. Adverse Events

\begin{tabular}{lc}
\hline Adverse events & Rifaximin-based quadruple therapy \\
\hline Nausea or vomiting & $2(7.4)$ \\
Diarrhea, abdominal pain & $1(3.7)$ \\
Dyspepsia & $3(11.1)$ \\
\hline
\end{tabular}

Values are presented as $\mathrm{n}(\%)$.

Table 3. Eradication Rates by Intention-to-treat and Per-protocol Analyses

\begin{tabular}{lc}
\hline & Rifaximin-based quadruple therapy \\
\hline ITT analysis & $70.4 \%(19 / 27)$ \\
Eradication rate & \\
PP analysis & $86.4 \%(19 / 22)$ \\
Eradication rate
\end{tabular}

ITT, intention-to-treat; PP, per-protocol. maining five patients were excluded from the PP analysis: four patients (14.7\%) were lost to follow-up and one patient $(3.7 \%)$ discontinued therapy owing to severe adverse events. Compliance with the therapy, that is, the percentage of patients who had taken $>85 \%$ of the drugs prescribed, was 96.3\% ( $\mathrm{n}=26$ ) (Fig. 1).

\section{Adverse events}

We also assessed the safety of the therapy and the associated adverse events. Six patients (22.2\%) experienced adverse events during treatment. Most of the adverse events were mild gastrointestinal problems; $7.4 \%(\mathrm{n}=2)$ experienced nausea or vomiting, 3.7\% $(n=1)$ had diarrhea and abdominal pain, and $11.1 \%(n=3)$ had dyspepsia. Only one patient complained of severe nausea and discontinued therapy. The adverse events are summarized in Table 2.

\section{H. pylori eradication rates and resistance rates to antibiotics}

The H. pylori eradication rates and resistance to antibiotics are described in Table 3 and 4, respectively. The ITT eradication rate was $70.4 \%$ (19/27), and the PP eradication rate was $86.3 \%$ (19/22). Owing to the various limitations of this study, $H$. pylori culture was successful for only three patients. Antibiotic resistance rates to amoxicillin, clarithromycin, metronidazole, rifaximin, and rifabutin were $0 \%, 66.7 \%, 66.7 \%, 66.7 \%$, and $0 \%$, respectively. Of the three patients who experienced antibiotic resistance testing, two were resistant to rifaximin, but there was no follow-up. Successful eradication occurred in one patient. In addition, none of these three

Table 4. Antibiotic Resistance in Three Patients Tested for Antibiotic Susceptibility

\begin{tabular}{|c|c|c|c|c|c|c|}
\hline & \multicolumn{5}{|c|}{ Minimal inhibitary concentration } & \multirow{2}{*}{$\begin{array}{c}\text { Eradication after } \\
\text { treatment }\end{array}$} \\
\hline & Amoxicillin $(\mathrm{R} \geq 0.5)$ & Clarithromycin $(\mathrm{R}>1)$ & Metronidazole $(\mathrm{R}>8)$ & Rifaximin $(\mathrm{R}>1)$ & Rifabutin $(\mathrm{R} \geq 0.25)$ & \\
\hline A & $\leq 0.125$ & $\leq 0.25$ & $4 \sim 8$ & No growth & $\leq 0.015$ & Follow-up loss \\
\hline B & $\leq 0.125$ & $16 \sim 32$ & $\geq 32$ & $2 \sim 4$ & $\leq 0.015$ & Follow-up loss \\
\hline $\mathrm{C}$ & $\leq 0.125$ & $\leq 0.25$ & $\geq 32$ & $0.25 \sim 0.5$ & $\leq 0.015$ & Yes \\
\hline
\end{tabular}

Breakpoints for resistance were defined as $0.5 \mathrm{mg} / \mathrm{L}$ for amoxicillin, $1 \mathrm{mg} / \mathrm{L}$ for clarithromycin, $8 \mathrm{mg} / \mathrm{L}$ for metronidazole, $1 \mathrm{mg} / \mathrm{L}$ for rifaximin, and $0.25 \mathrm{mg} / \mathrm{L}$ for rifabutin. 
patients were resistant to rifabutin.

\section{DISCUSSION}

Although several $H$. pylori eradication regimens have been recently developed, there is no perfect treatment for $H$. pylori eradication, and an eradication rate of $100 \%$ has not been reported. ${ }^{26}$ Although there are a variety of factors that account for the inefficiency of the eradication regimens, it is most likely due to the adverse effects caused by the antibiotics, resulting in poor compliance to treatment, and increased antibiotic resistance to clarithromycin and metronidazole. ${ }^{27,28}$ Rifamycin derivatives (rifampicin, rifabutin, and rifaximin) exert antibacterial activity against $H$. pylori. Rifabutin has been used as a rescue therapy after failed eradication therapy. Rifaximin is a relatively new alternative antibiotic, belonging to the same family as rifabutin; it is poorly absorbed in the gastrointestinal tract, remains highly concentrated within the gastrointestinal tract, and does not cause adverse effects. ${ }^{29,30}$ Owing to these pharmacokinetic and pharmacodynamic characteristics, the activity of rifaximin against $H$. pylori was studied in vitro and in vivo. In an in vitro study, 43 strains of $H$. pylori were examined to determine MICs using four antimicrobial compounds: rifaximin, rifampicin, amoxicillin, and colloidal bismuth subcitrate. ${ }^{31}$ The activity of rifaximin was intermediate, between that of amoxicillin, one of the most active agents against $H$. pylori, and colloid bismuth subcitrate, which has moderate activity. ${ }^{32}$ Interestingly, the MIC value of rifaximin is hardly affected by lowering the $\mathrm{pH}$ from 7.2 to 6 . No strain exhibited primary resistance to rifaximin. ${ }^{31}$ So far, several studies have assessed the efficacy of rifaximin, focusing on dual, triple, and quadruple regimens. As rifaximin is known to be effective against H. pylori strains resistant to clarithromycin, it was supposed that this antibiotic could be associated with this drug in vivo. ${ }^{21,33}$ Several studies evaluating the efficacy of rifaximin have been performed, mostly with dual or triple therapies. Dual regimens combining omeprazole or clarithromycin with metronidazole or bismuth salts have shown eradication rates between $40 \%$ and $70 \%{ }^{34,35}$ Triple regimens com- bining rifaximin suspension (600 mg t.i.d.), omeprazole (40 mg o.d.), and amoxicillin (1 g b.i.d.) for 2 weeks achieved eradication rates of $60 \%$. Gasbarrini et al. ${ }^{22}$ chose the triple therapy combining rifaximin ( $400 \mathrm{mg}$ t.i.d.), esomeprazole (40 mg o.d.), and clarithromycin (500 mg b.i.d.) for 7 days and obtained an eradication rate of $58 \%{ }^{36}$ Two papers have been published about rifaximin-based quadruple therapies. One (omeprazole 20 mg o.d., amoxicillin 1 g b.i.d., rifaximin 400 mg b.i.d., and levofloxacin $200 \mathrm{mg}$ b.i.d. for 7 days) describes a double-blind, randomized, controlled trial that obtained an eradication rate of $80 \%{ }^{23}$ The other included a quadruple therapy with rifaximin (rifaximin $400 \mathrm{mg}$ t.i.d., clarithromycin $500 \mathrm{mg}$ t.i.d., amoxicillin $1 \mathrm{~g}$ b.i.d., and omeprazole $20 \mathrm{mg}$ for 10 days) with a PP eradication rate of only $61 \%$. In our study, we combined rifaximin with clarithromycin, amoxicillin, and PPI, and found a PP eradication rate of $86.3 \%$, slightly below the established efficacy consensus threshold (90\%). This study has demonstrated the considerable efficacy of the rifaximin-based quadruple therapy and its remarkable safety. However, this regimen cannot be recognized as one of the standard eradication regimens. Because our study employed only three resistance tests, it is difficult to draw firm conclusions. Although it is true that clarithromycin has high resistance potential, the high resistance rate observed in our study was thought to be because of the small number of samples tested for resistance. However, resistance to rifaximin is 66.6\%; therefore, it is likely that rifaximin resistance is quite high in Korea. Unlike rifaximin, the resistance rate to rifabutin was $0 \%$, and it can be used in the rescue therapy for $H$. pylori. The reason rifabutin is useful in $H$. pylori treatment is that rifabutin has no cross-resistance with clarithromycin and is not a widely used antibiotic. Therefore, it is considered that $H$. pylori has a low resistance to rifabutin. ${ }^{37}$ The results of our study show the practical limitations of first-line rifaximin based quadruple therapy, and further research on third-line rifabutin-based regimens is needed.

In conclusion, the results of this study have described the limitations of current rifaximin-based quadruple therapy and the potential for a rifabutin-based rescue regimen in Korea. Further studies exploring different 
drug combinations, dosing, and durations of therapy are necessary to verify the efficacy of rifaximin and rifabutin in the treatment of $H$. pylori infection.

\section{CONFLICT OF INTEREST}

No potential conflict of interest relevant to this article was reported.

\section{ORCID}

\begin{tabular}{|c|c|}
\hline Kim & \\
\hline ruk Yoon & (iD https://orcid.org/0000-0002-2657-0349 \\
\hline Jun Oh & /orcid.org/0000-0002- \\
\hline & A \\
\hline n Jin Choi & $2-1922-93$ \\
\hline 1 Min Shin & ttps://orcid.org/0000-0003-2265- \\
\hline o Park & ttps://orcid.org/0000-0003-1893- \\
\hline & $\mathrm{cg} / 0000-0002-939$ \\
\hline & $\mathrm{rg} / 0000-0002-637$ \\
\hline
\end{tabular}

\section{REFERENCES}

1. Qadri Q, Rasool R, Gulzar GM, Naqash S, Shah ZA. H. pylori infection, inflammation and gastric cancer. J Gastrointest Cancer 2014; $45: 126-132$.

2. Yari F, Abiri R, Aryan E, Ahmadi Jouybari T, Navabi J, Alvandi A. Loop-mediated isothermal amplification as a fast noninvasive method of Helicobacter pylori diagnosis. J Clin Lab Anal 2016;30:464-470.

3. Du LJ, Chen BR, Kim JJ, Kim S, Shen JH, Dai N. Helicobacter pylori eradication therapy for functional dyspepsia: systematic review and meta-analysis. World J Gastroenterol 2016;22:3486-3495.

4. Zhang RG, Duan GC, Fan QT, Chen SY. Role of Helicobacter pylori infection in pathogenesis of gastric carcinoma. World J Gastrointest Pathophysiol 2016;7:97-107.

5. Gasbarrini A, Carloni E, Gasbarrini G, Chisholm SA. Helicobacter pyloriand extragastric diseases--other Helicobacters. Helicobacter 2004;9 Suppl 1:57-66.

6. Vázquez Romero M, Bermejo San José F, Boixeda de Miquel D, et al. Chronic urticaria and Helicobacter pylori. Med Clin (Barc) 2004;122:573-575.

7. Franchini M, Veneri D. Helicobacter pylori infection and immune thrombocytopenic purpura: an update. Helicobacter 2004;9:342-346.

8. Candelli M, Carloni E, Nista EC, et al. Helicobacter pylori eradication and acne rosacea resolution: cause-effect or $\mathrm{CO}^{-}$ incidence? Dig Liver Dis 2004;36:163.
9. Gabrielli M, Fiore G, Candelli M, et al. Re: "Chronic Helicobacter pylori infection and migraine: a case-control study" (Pinessi L, Savi L, Pellicano R, et al. Headache. 2000;40:836-839). Headache 2002;42:236-237; author reply 235-236.

10. Gasbarrini A, Gabrielli M, Fiore G, et al. Association between Helicobacter pylori cytotoxic type I CagA-positive strains and migraine with aura. Cephalalgia 2000;20:561-565.

11. Malfertheiner P, Megraud F, O'Morain C, et al. Current concepts in the management of Helicobacter pylori infection: the Maastricht III Consensus Report. Gut 2007;56:772-781.

12. Chey WD, Wong BC; Practice Parameters Committee of the American College of Gastroenterology. American College of Gastroenterology guideline on the management of Helicobacter pylori infection. Am J Gastroenterol 2007;102:1808-1825.

13. Kim JM, Kim JS, Jung HC, Kim N, Kim YJ, Song IS. Distribution of antibiotic MICs for Helicobacter pyloristrains over a 16-year period in patients from Seoul, South Korea. Antimicrob Agents Chemother 2004; 48:4843-4847.

14. Kim N, Kim JM, Kim CH, et al. Institutional difference of antibiotic resistance of Helicobacter pylori strains in Korea. J Clin Gastroenterol 2006;40:683-687.

15. Bock H, Koop H, Lehn N, Heep M. Rifabutin-based triple therapy after failure of Helicobacter pylori eradication treatment: preliminary experience. J Clin Gastroenterol 2000;31:222-225.

16. Perri F, Festa V, Clemente R, Quitadamo M, Andriulli A. Rifabutin-based 'rescue therapy' for Helicobacter pylori infected patients after failure of standard regimens. Aliment Pharmacol Ther 2000;14:311-316.

17. Canducci F, Ojetti V, Pola P, Gasbarrini G, Gasbarrini A. Rifabutin-based Helicobacter pylori eradication 'rescue therapy'. Aliment Pharmacol Ther 2001;15:143.

18. Scarpignato C. Rifaximin: a poorly absorbed antibiotic. Pharmacology and clinical use. Basel: Karger Medical and Scientific Publishers, 2005.

19. Jiang ZD, DuPont HL. Rifaximin: in vitro and in vivo antibacterial activity--a review. Chemotherapy 2005;51 Suppl 1: 67-72.

20. Quesada M, Sanfeliu I, Junquera F, Segura F, Calvet X. Evaluation of Helicobacter pylori susceptibility to rifaximin. Gastroenterol Hepatol 2004;27:393-396.

21. Scarpignato C, Pelosini I. Bismuth compounds for eradication of Helicobacter pylori: pharmacology and safety. In: Scarpignato C, Bianchi Porro G, eds. Clinical pharmacology and therapy of Helicobacter pylori infection. Vol. 11. Basel: Karger Publishers, 1999:87-127.

22. Gasbarrini A, Lauritano EC, Nista EC, et al. Rifaximin-based regimens for eradication of Helicobacter pylori: a pilot study. Dig Dis 2006;24:195-200.

23. Choi KH, Chung WC, Lee KM, et al. Efficacy of levofloxacin and rifaximin based quadruple therapy in Helicobacter pyloriassociated gastroduodenal disease: a double-blind, randomized controlled trial. J Korean Med Sci 2011;26:785-790.

24. Lim HC, Lee YJ, An B, Lee SW, Lee YC, Moon BS. Rifabutinbased high-dose proton-pump inhibitor and amoxicillin triple 
regimen as the rescue treatment for Helicobacter pylori. Helicobacter 2014;19:455-461.

25. Zhang YX, Zhou LY, Song ZQ, Zhang JZ, He LH, Ding Y. Primary antibiotic resistance of Helicobacter pyloristrains isolated from patients with dyspeptic symptoms in Beijing: a prospective serial study. World J Gastroenterol 2015;21:2786-2792.

26. Nizhevich AA, Shcherbakov PL, Akhmadeeva ÉN, Khasanov RSh. Rifaximin in combined treatment of the Helicobacter $p y^{-}$ lori infection in childhood. Eksp Klin Gastroenterol 2011;1: 85-87.

27. Gisbert JP, Calvet X. Review article: the effectiveness of standard triple therapy for Helicobacter pylori has not changed over the last decade, but it is not good enough. Aliment Pharmacol Ther 2011;34:1255-1268.

28. Houben MH, van de Beek D, Hensen EF, de Craen AJ, Rauws EA, Tytgat GN. A systematic review of Helicobacter pylori eradication therapy--the impact of antimicrobial resistance on eradication rates. Aliment Pharmacol Ther 1999;13:1047-1055.

29. Scarpignato C, Pelosini I. Rifaximin, a poorly absorbed antibiotic: pharmacology and clinical potential. Chemotherapy 2005;51 Suppl 1:36-66.

30. Baker DE. Rifaximin: a nonabsorbed oral antibiotic. Rev Gastroenterol Disord 2005;5:19-30.
31. Mégraud F, Bouffant F, Camou Juncas C. In vitro activity of rifaximin against Helicobacter pylori. Eur J Clin Microbiol Infect Dis 1994;13:184-186.

32. De Giorgio R, Stanghellini V, Barbara G, Guerrini S, Ferrieri A, Corinaldesi R. Rifaximin and Helicobacter pylori eradication. Eur Rev Med Pharmacol Sci 1997;1:105-110.

33. Lauritano EC, Gabrielli M, Lupascu A, et al. Rifaximin dose-finding study for the treatment of small intestinal bacterial overgrowth. Aliment Pharmacol Ther 2005;22:31-35.

34. Dell'Anna A, Azzarone P, Ferrieri A. A randomized openly comparative study between rifaximin suspension versus rifaximin pills for the eradication of Helicobacter pylori. Eur Rev Med Pharmacol Sci 1999;3:105-110.

35. Pretolani S, Bonvicini F, Brocci E, et al. Effect of rifaximin, a new non-absorbed antibiotic, in the treatment of Helicobacter pylori infection. Acta Gastroenterol Belg 1993;56:144A.

36. Vaira D, Menegatti M, Miglioli M, et al. Rifaximin suspension for the eradication of Helicobacter pylori. Curr Ther Res 1997;58: 300-308.

37. Mégraud F, Lamouliatte H. Review article: the treatment of refractory Helicobacter pylori infection. Aliment Pharmacol Ther 2003;17:1333-1343. 